\title{
Conformational adaptation and manipulation of manganese tetra(4-pyridyl)porphyrin molecules on $\mathrm{Cu}(111)$
}

Xianwen Chen, Shulai Lei, Christian Lotze, Constantin Czekelius, Beate Paulus, and Katharina J. Franke

Citation: The Journal of Chemical Physics 146, 092316 (2017); doi: 10.1063/1.4974313

View online: http://dx.doi.org/10.1063/1.4974313

View Table of Contents: http://aip.scitation.org/toc/jcp/146/9

Published by the American Institute of Physics

\section{Articles you may be interested in}

From tunneling to contact in a magnetic atom: The non-equilibrium Kondo effect

The Journal of Chemical Physics 146, 092309092309 (2016); 10.1063/1.4972874

Spin crossover in Fe(phen)2(NCS)2 complexes on metallic surfaces

The Journal of Chemical Physics 146, 092312092312 (2017); 10.1063/1.4973511

Forces from periodic charging of adsorbed molecules

The Journal of Chemical Physics 146, 092327092327 (2017); 10.1063/1.4975607

Temperature dependent tunneling conductance of single molecule junctions

The Journal of Chemical Physics 146, 092311092311 (2017); 10.1063/1.4973318

Kondo behavior and conductance through $3 \mathrm{~d}$ impurities in gold chains doped with oxygen

The Journal of Chemical Physics 146, 092315092315 (2017); 10.1063/1.4973982

A tunable electronic beam splitter realized with crossed graphene nanoribbons

The Journal of Chemical Physics 146, 092318092318 (2017); 10.1063/1.4974895

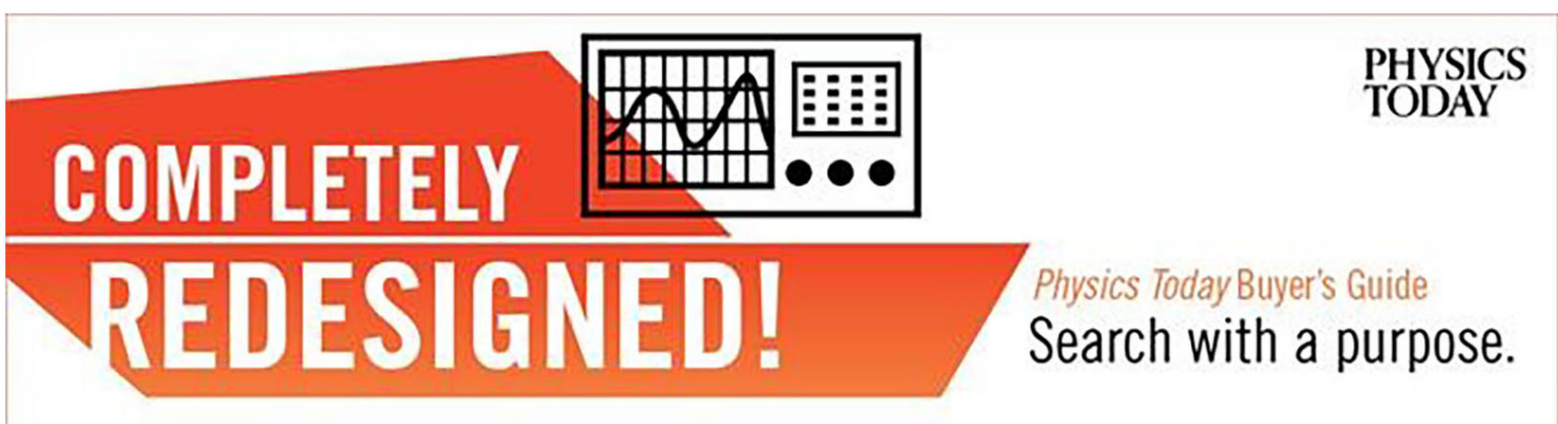




\title{
Conformational adaptation and manipulation of manganese tetra(4-pyridyl)porphyrin molecules on $\mathrm{Cu}(111)$
}

\author{
Xianwen Chen, ${ }^{1}$ Shulai Lei, ${ }^{2}$ Christian Lotze, ${ }^{1}$ Constantin Czekelius, ${ }^{3}$ Beate Paulus, ${ }^{2}$ \\ and Katharina J. Franke ${ }^{1}$ \\ ${ }^{1}$ Fachbereich Physik, Freie Universität Berlin, Arnimallee 14, 14195 Berlin, Germany \\ ${ }^{2}$ Institut für Chemie und Biochemie, Freie Universität Berlin, Takustraße 3, 14195 Berlin, Germany \\ ${ }^{3}$ Institut für Organische Chemie und Makromolekulare Chemie, Heinrich-Heine Universität Düsseldorf, \\ Universitätsstraße 1, 40225 Düsseldorf, Germany
}

(Received 31 October 2016; accepted 6 January 2017; published online 24 January 2017)

Porphyrins are highly flexible molecules and well known to adapt to their local environment via conformational changes. We studied the self-assembly of manganese meso-tetra(4-pyridyl)porphyrin (Mn-TPyP) molecules on a $\mathrm{Cu}(111)$ surface by low temperature scanning tunneling microscopy (STM) and atomic force microscopy (ATM). We observe molecular chains along the $\langle 1 \overline{1} 0\rangle$ direction of the substrate. Within these chains, we identify two molecular conformations, which differ by the orientation of the upward bending of the macrocycle. Using density functional theory, we show that this saddle shape is a consequence of the rotation and inclination of the pyridyl groups towards $\mathrm{Cu}$ adatoms, which stabilize the metal-organic chains. The molecular conformations obey a strict alternation, reflecting the mutual enforcement of conformational adaptation in densely packed structures. Tunneling electrons from the STM tip can induce changes in the orientation of the pyridyl endgroups. The switching behaviour varies with the different adsorption configurations. Published by AIP Publishing. [http://dx.doi.org/10.1063/1.4974313]

\section{INTRODUCTION}

Porphyrins and metalloporphyrins are versatile species for constructing supramolecular architectures on metal surfaces via non-covalent intermolecular interactions ${ }^{1-5}$ or metalcoordination bonds. ${ }^{4-11}$ In these assemblies, the molecules can take different conformations as a result of an adaptation to the substrate lattice periodicity as well as an optimization of the bonding energy to lateral neighbors (atoms or molecules). ${ }^{12-15}$ These interactions can be steered by the design of the molecular endgroups. Famous examples are terminations with lonepair electrons, e.g., pyridyl or cyanobenzene, which are prone to form coordination bonds with transition metal atoms. These interactions drive the formation of metal-organic networks with co-adsorbed atoms as well as with substrate adatoms. ${ }^{16-19}$ The energy gain in bond formation is partially consumed by molecular deformations, such as a distortion of the macrocycle and rearrangements of the terminal substitutes. The resulting molecular structures are often referred to as saddle or dome conformers. ${ }^{20-22}$

The structural flexibility promotes porphyrins as promising candidates for molecular switches, which undergo conformational changes by external stimuli. The first great promise arose from the reversible rotation of a di-butylphenyl leg in individual copper meso-tetrakis(3,5-di-tertbutyl-phenyl)porphyrin (Cu-TBPP) molecules on a $\mathrm{Cu}(211)$ surface by manipulation with a scanning tunneling microscopy (STM) tip. ${ }^{23}$ Several examples of STM-induced switching between different conformations of the macrocycle have since been achieved, such as the interconversion between two nonplanar conformations of single $\mathrm{Zn}$ (II) etioporphyrin I molecules adsorbed on a $\mathrm{NiAl}(110)$ surface $^{24}$ or the switching between the concave and convex conformations of 2H-meso-tetrakis(3,5-di-tert-butyl-phenyl)porphyrin (2HTTBPP) molecules in self-assembled rows on a $\mathrm{Cu}(111)$ surface. Whereas the latter was non-reversible with the STM tip, it did show temperature-induced reversible switching. ${ }^{25}$ Direct evidence of temperature and STM-induced switching between different saddle shapes of individual 2H-tetra-phenylporpyhrin (TPP) and CuTPP molecules has recently been obtained by detailed height-dependent STM and atomic force microscopy (AFM) measurements. ${ }^{26}$

Some concepts of molecular electronics envision the use of single molecules as switches. Porphyrins may act as such switches when contacted by two electrodes. Their conformational changes are naturally accompanied by changes in the conductance between the electrodes. The implementation of such concepts is still in its infancy. To date, the control of the states is neither sufficiently reliable nor suitable for upscaling or room temperature applications. Up-scaling methods require a design strategy for addressable arrays of molecules. One approach includes the self-assembly of molecules. The assembly process relies on intermolecular interactions, which determine the structure of the resulting layers. These interactions, then again, may modify the electronic structure, conformation, or switching ability of the porphyrin. Hence, it is necessary to gain an understanding of the molecular interactions driving the formation of porphyrin assemblies, how they affect the molecular conformation and electronic structure, and if conformational changes can be induced in a reliable fashion.

Here, we combine low temperature scanning tunneling spectroscopy, atomic force microscopy, and density functional theory (DFT) to elucidate on the structural and electronic 
properties of manganese meso-tetra(4-pyridyl)porphyrin (Mn-TPyP) on a $\mathrm{Cu}(111)$ surface. We find self-assembled molecular chains with two distinct conformers. Our multitechnique approach reveals that the structure is a result of $\mathrm{Cu}$ adatoms being incorporated into the chains in agreement with earlier observations of free-base TPyP molecules. ${ }^{16,19}$ The bonding configuration to the $\mathrm{Cu}$ adatoms imposes a strict rule of alternation to the molecular conformers along the chain. Neighboring molecules with the same conformation are sterically suppressed and are only allowed if a second $\mathrm{Cu}$ atom is included as additional spacer. Despite the dense bonding configuration in the alternating chains, we were able to induce reversible rotations of the pyridyl endgroups by tunneling electrons. In contrast to this, we only find an irreversible upwards rotation of the pyridyl legs in the chains with two $\mathrm{Cu}$ adatoms between the molecules.

\section{EXPERIMENTAL AND THEORETICAL DETAILS}

All experiments were carried out under ultra-high vacuum conditions. The $\mathrm{Cu}(111)$ sample was cleaned by standard sputtering-annealing cycles. Mn-TPyP-acetate molecules (Fig. 1) were evaporated from a Knudsen cell held at $470 \mathrm{~K}$ onto the $\mathrm{Cu}(111)$ surface at room temperature. Post-deposition annealing was done at $410 \mathrm{~K}$ in order to ensure reductive cleavage of the acetate ligand and to allow for self-assembly of the manganese(II) complex on the surface. The sample was
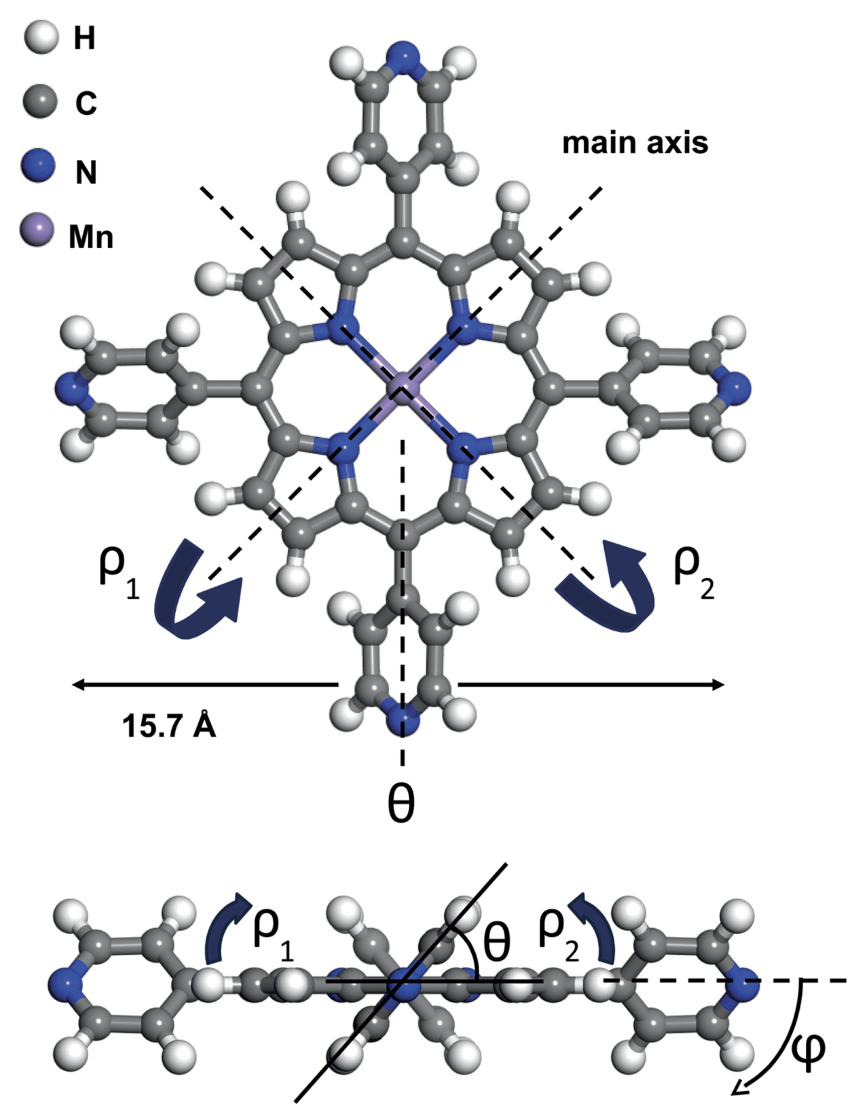

FIG. 1. Conformational flexibility of the Mn-TPyP molecule. The molecule consists of a macrocycle plane with a central $\mathrm{Mn}$ atom and 4 peripheral pyridyl end groups. $\rho_{1,2}$ represent the deformation angle of pyrrole rings in the macrocycle. $\theta$ and $\phi$ represent the rotation and bending orientation of meso-pyridyl relative to the molecular core, respectively. then cooled down and transferred into a CreaTec scanning tunneling microscope (STM)/atomic force microscope (AFM) with a base temperature of $4.6 \mathrm{~K}$. The microscope is equipped with a qPlus tuning fork sensor for combined measurements of tunneling current and frequency shift. The presented STM images were recorded in constant-current mode. The maps of the frequency shift were acquired at constant height. Differential conductance spectra were acquired at constant current, i.e., with activated feedback-loop, using a lock-in amplifier.

For the computational approach, the $\mathrm{Cu}(111)$ surface was modeled by a three-layer slab. The lattice constant of $\mathrm{Cu}$ was fixed to the bulk value of $3.63 \AA .{ }^{27} \mathrm{Mn}$-TPyP molecules were placed at different adsorption sites on the surface to determine the energetically most favorable site. The top, bridge, hollowhcp and hollow-fcc sites are defined with respect to the Mn on $\mathrm{Cu}$. The periodic supercells were chosen in accordance with the experimentally determined molecular distances and assuming a lattice commensurability of $15.43 \AA$ along a densepacked row in $\langle 1 \overline{1} 0\rangle$ direction. The distance between the rows was chosen as $22.25 \AA$. The size of the supercell in the direction perpendicular to the metal surface was set to $25 \AA$ to avoid the interaction between neighboring slabs.

Spin-polarized electronic structure and geometric relaxation calculations were performed by using the projector augmented wave pseudopotentials as implemented in the Vienna $A b$ initio simulation package (VASP). ${ }^{28,29}$ Exchange correlation interactions are described by the PerdewBurke-Ernzerhof $(\mathrm{PBE})^{30}$ generalized gradient approximation (GGA). Recently, it was shown that van der Waals interactions play a decisive role in the adsorption of free-base porphyrin molecules on metal surfaces. ${ }^{15}$ In order to explore the effects of van der Waals interactions of Mn-TPyP on $\mathrm{Cu}(111)$, we also carried out $\mathrm{PBE}+\mathrm{D} 2^{31}$ calculations. GGA in general cannot properly describe the on-site Coulomb interaction of $3 d$ electrons. Therefore, we carried out $\mathrm{PBE}+\mathrm{U}$ calculations by choosing a correlation energy $\mathrm{U}$ of $4.2 \mathrm{eV}$ and an exchange energy $\mathbf{J}$ of $1 \mathrm{eV}$ for $\mathrm{Mn} d$ orbitals. ${ }^{32}$ The Brillouin-zone integration was performed at the $\Gamma$-point only due to the large size of the examined supercell. The plane wave kinetic energy cutoff was set to be $400 \mathrm{eV}$. Atomic positions were optimized until the atomic forces were smaller than $0.01 \mathrm{eV} / \AA$. All adsorption energies were determined from full structure optimization of Mn-TPyP, keeping all the metal substrate layers fixed.

\section{RESULTS AND DISCUSSION}

\section{A. Self-assembled chains of Mn-TPyP on $\mathrm{Cu}(111)$}

Deposition of Mn-TPyP at room temperature and posterior annealing to $410 \pm 10 \mathrm{~K}$ leads to the formation of molecular chains along the $\langle 1 \overline{1} 0\rangle$ direction of the $\mathrm{Cu}(111)$ substrate (Figs. 2(a) and 2(b)). Occasionally, we also observe honeycomb structures with their molecular segments aligned along the same surface directions. Such arrangements have been observed for free-base TPyP on a $\mathrm{Cu}(111)$ surface. ${ }^{16} \mathrm{We}$ identify two types of molecules within the chains: there is a "dark" configuration, which we label as " $\mathrm{A}$ " in the following. These molecules appear almost featureless. Their apparent 

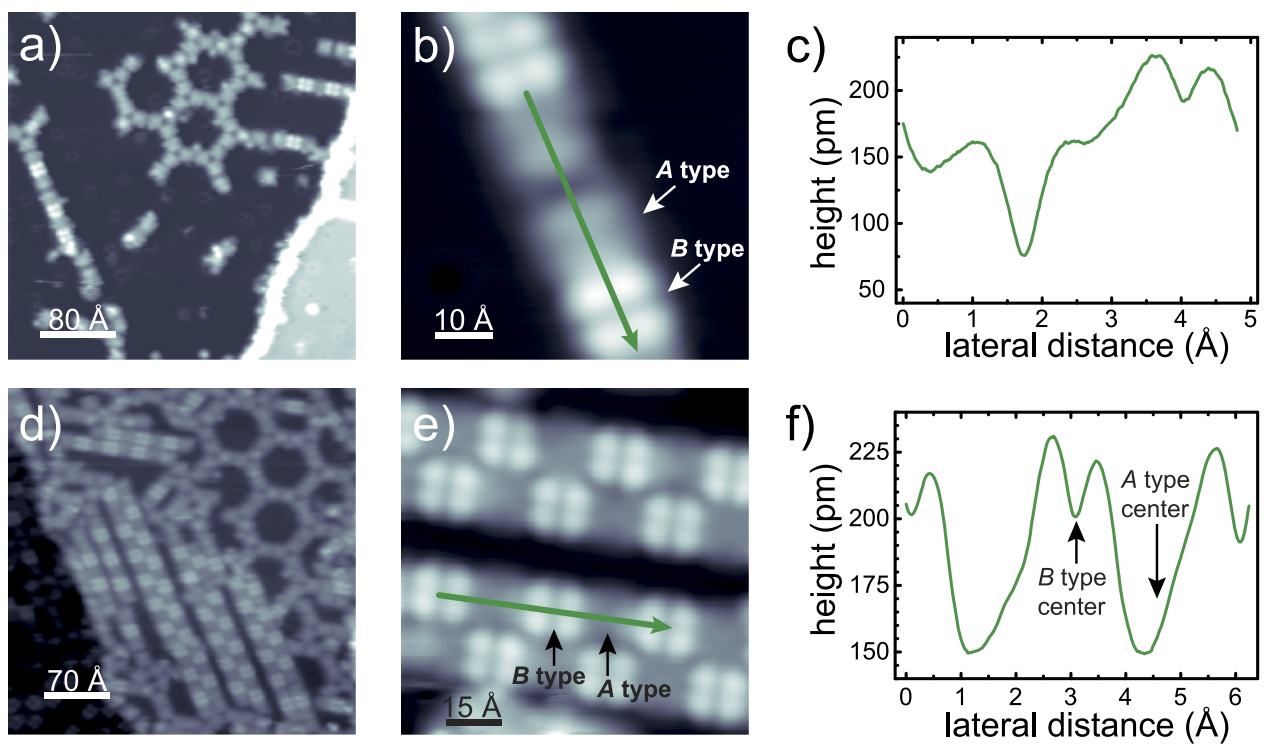

FIG. 2. Self-assembly of Mn-TPyP molecules on a Cu(111) surface. (a) Constant-current STM overview and (b) close-up view of Mn-TPyP chain. Dark (type A) and bright (type B) molecules irregularly arrange in mixed chains and hexagonal networks. The molecular chains are aligned along $\langle 1 \overline{1} 0\rangle$ directions. (d) Constant-current STM overview and (e) close-up view of Mn-TPyP molecules at larger coverage. Single- or multiple-row chains consist of alternating bright (type B) and dark (type A) molecules and hexagonal networks. (c) and (f) Line-profiles along the chains as indicated in the images. The height of the Cu surface is set to zero. Tunneling conditions: $V=1.0 \mathrm{~V}$ and $I=100 \mathrm{pA}$.

height is $1.5 \AA$ (at $V=1.0 \mathrm{~V}$ ). The "brighter" molecules consist of four lobes with an apparent height of $2 \AA$ (see Figs. 2(c) and 2(f)) and will be labeled "B" henceforth. By superimposing the molecular structure model of Mn-TPyP onto the STM image, we assign each lobe to one of the pyridyl rings. The distance between type A and B molecules is $14.6 \pm 0.5 \AA$. Type A molecules may lie adjacent to each other, but then obey a distance of $19.4 \pm 0.5 \AA$. We never observe type B molecules adjacent to each other. Increasing the coverage at the same annealing temperature enforces the molecular chains to be strictly alternating with an A-B periodicity. Moreover, neighboring chains are shifted by one molecular unit, such that a checkerboard structure appears (Figs. 2(d) and 2(e)). The apparent heights of the molecules are the same as in the single-molecule chains. We therefore also assign them to types A and B. The mixed structure of type A and type $\mathrm{B}$ molecules seems to be the thermodynamically most stable structure.

The large distance between the molecules compared to their gas phase size of $\sim 11 \AA$ suggests the incorporation of $\mathrm{Cu}$ atoms between the molecules. This has also been suggested for the free-base TPyP molecules at a distance of $\sim 15.5 \AA$ by Klappenberger and co-workers on the very same surface. ${ }^{16}$ The attraction of $\mathrm{Cu}$ atoms from diffusing atoms during the annealing process is ascribed to the large affinity of the lonepair electrons of the terminal pyridyl unit towards transition metal atoms. ${ }^{8,33,34}$ The larger molecular distance in the pure type A chains is compatible with the inclusion of two adatoms. Indeed, we corroborate this assumption by STM measurements with a functionalized tip. Figure 3(a) reveals two pairs of protrusions between the type A molecules. However, we do not have direct evidence of the atoms between type A and B molecules. Metal adatoms in metal-coordinated supramolecular structures are difficult to be resolved in STM images due to electronic effects. ${ }^{35-42}$ Moreover, the close molecular proximity may not allow for the STM tip to approach sufficiently close to the adatoms. Therefore, only larger bonding motifs with two or more $\mathrm{Cu}$ atoms linking organic moieties were observed for ordered patterns on surfaces. ${ }^{16,43,44}$ We thus conclude that the protrusions in the STM images correspond to two $\mathrm{Cu}$ atoms between the pyridyl moieties of neighboring type $\mathrm{A}$ molecules.

a)

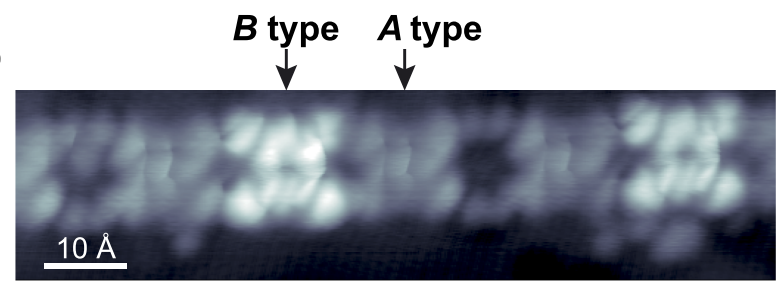

b)

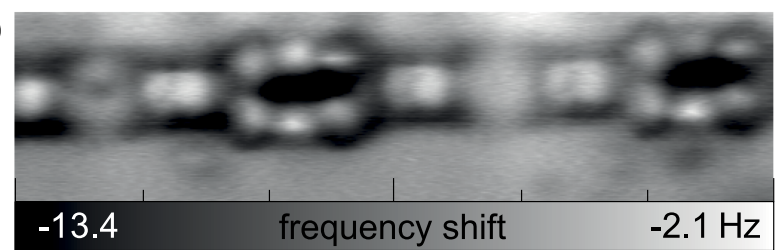

c)

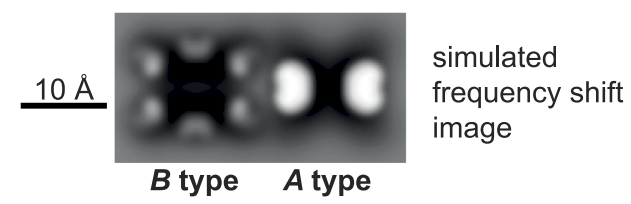

FIG. 3. High-resolution STM and AFM images for a mixed-phase molecular chain measured with a CO-functionalized tip at constant height. The height is given by the tunneling current $I=110 \mathrm{pA}$ at a bias voltage $V=0.1 \mathrm{~V}$ over the bare $\mathrm{Cu}$ surface. (a) Current image at the given setpoint. (b) Frequency-shift image on the same molecular chain at $\Delta z=0.5 \AA$ retracted from the setpoint. The tip probes the repulsive regime at this height. (c) Simulated AFM image using the minimized structure from DFT. 


\section{B. Molecular conformation}

The drastically different appearance of type A and B molecules in the STM images suggests a significantly different conformation accompanied by a different electronic structure. To gain further insights into the conformations, we carry out simultaneous STM and AFM measurements with a tuning fork sensor. We functionalize the tip with a $\mathrm{CO}$ molecule for better lateral resolution. This procedure has been frequently employed for imaging intramolecular structures because the tip can be brought into close proximity of the molecular layer without significant perturbation. ${ }^{45-47}$

We show a typical frequency-shift image at constant height in the repulsive regime in Fig. 3(b). The bright contrast indicates the strongest tip-sample repulsion and can be correlated with the largest molecular height above the surface. Whereas the image of the tunneling current (Fig. 3(a)) suggests fourfold symmetric molecules, the AFM signal (Fig. 3(b)) clearly shows that both types of molecules are distorted and exhibit a reduced twofold symmetry. Porphyrin molecules are well known to undergo rotations and inclinations of the meso-substituents as well as a bending of the macrocycle. ${ }^{16}$ The observed two-lobe structure of type A molecules suggests a strong upward bending of the macrocycle along the molecular chain axis. In type B molecules, the upward bending of the macrocycle occurs perpendicular to the chain direction. Here, the pyridyl rings contribute to the AFM signal with approximately the same height.

To corroborate and quantify the molecular conformations, we carry out DFT calculations. We use four different angles to describe the geometry of porphyrins. $\rho_{1,2}$ represent the deformation angles of the macrocycle parallel and perpendicular to the molecular rows, respectively. $\theta$ and $\phi$ describe the rotation and bending of the meso-substitutes with respect to the molecule's main plane (see Fig. 1).

Whereas the experiment provides the molecular orientation with respect to the $\mathrm{Cu}$ lattice, it does not specify the adsorption site. Hence, we first explore the adsorption conformations and energies for molecules with the Mn center sitting on top, bridge, hollow-hcp and hollow-fcc sites of the substrate. We thus place a molecule, which has been relaxed in gas phase, on these four sites and let it fully relax on a structurally fixed $\mathrm{Cu}(111)$ surface. We find two energetic minima for each adsorption site: one with an upward bent macrocycle along the $\langle 1 \overline{1} 0\rangle$ direction and another one with the macrocycle bent upwards along the $\langle 111\rangle$ direction. We identify these as the type A and type B molecules observed in experiment, respectively. The adsorption energies are summarized in Table I. They are very similar for the two conformers and vary only slightly for the different adsorption sites. This suggests that the largest

TABLE I. Adsorption energies of type A and B Mn-TPyP on different sites of $\mathrm{Cu}(111)$ obtained from $\mathrm{PBE}+\mathrm{D} 2+\mathrm{U}$. Energies are given in $\mathrm{eV}$.

\begin{tabular}{lcccc}
\hline \hline & Top & Bridge & Hollow-hcp & Hollow-fcc \\
\hline A & -4.94 & -4.89 & -4.86 & -4.86 \\
B & -4.67 & -5.10 & -4.96 & -4.94 \\
\hline \hline
\end{tabular}

contribution to the adsorption energy stems from van der Waals interactions, which are not very sensitive to the precise adsorption site. A similar conclusion had been drawn for free-base tetra(4-bromophenyl)-porphyrin molecules on a $\mathrm{Cu}(111)$ surface. ${ }^{15}$ As a result of physisorption on the surface, the pyridyl legs nestle up against the surface, leading to an inclination angle $\theta=31.6^{\circ}$ for type A and $28.1^{\circ}$ for type B. Steric repulsion then forces the macrocycle to bend upwards, yielding the often-observed saddle shape configuration with a deformation angle $\rho_{1}=23.2^{\circ}$ for type A and $-15.3^{\circ}$ for type B along the molecular chain direction, while $\rho_{2}=-13.9^{\circ}$ for type A and $26.2^{\circ}$ for type $\mathrm{B}$ along the perpendicular direction to molecular chain.

Next, we adapt our calculations to the experimental findings, which suggest the bonding to $\mathrm{Cu}$ adatoms at the terminal pyridyl groups. Because we did not find significantly different adsorption energies for the Mn-TPyP in different lattice sites, we construct the metal-organic chains with the topsited molecules, which allow the $\mathrm{Cu}$ adatoms to be placed on the energetically preferred hollow sites. This configuration matches the experimental periodicity and orientation of the chains. The optimized model structures (three layers of $\mathrm{Cu}$ are fixed) are depicted in Figs. 4(a) and 4(b) for type A and type $\mathrm{B}$, respectively. For the type $\mathrm{A}$ adsorption, the $\mathrm{Cu}$ adatom is slightly shifted towards the surface $(0.08 \AA)$ compared to the ideal position on the plain surface, and for type B, the distance is slightly increased by $0.04 \AA$. For the mixed type A-type B adsorption, it stays nearly in the ideal position (Table II).

We observe that the adsorption energy of type A molecules in the metal-coordinated chains is increased by about $2.2 \mathrm{eV}$ compared to the non-coordinated case. Considering the bending of the pyridyl legs towards the $\mathrm{Cu}$ adatom, the inclination angle is reduced to $\theta=13.2^{\circ}$ for type A. The observation of the typical $\mathrm{Cu}-\mathrm{N}$ bond length of $2.12 \AA$ leads us to the conclusion that now a significant contribution to the adsorption energy stems from the metal-pyridyl bond. Accompanied by the pyridyl bending is a distortion of the macrocycle, leading to a pronounced saddle shape where the difference in the bending angles between the main axis and perpendicular is increased to $55.4^{\circ}$. Type $\mathrm{B}$ molecules also yield a strong binding to the $\mathrm{Cu}$ adatoms $(\mathrm{Cu}-\mathrm{N}$ bond length of $2.14 \AA$ ) but the geometrical changes are negligible. The inclination angle stays nearly unchanged $\left(\theta=28.8^{\circ}\right)$ and the bending of the macrocycle remains small (see values in Table III). Thereby also the adsorption height of the molecules stays as large as without adatoms, overall leading to a smaller gain in adsorption energy upon coordination. Hence, we conclude that type A molecules are favored in the metal-organic chains as compared to type B molecules. This is in agreement with the observation of pure type A chains, but no pure type B chains. However, we note that the experimentally observed neighboring type A molecules required the inclusion of a second $\mathrm{Cu}$ adatom. This points us to some repulsive contribution in pure type $\mathrm{A}-\mathrm{Cu}$ chains that is energetically outweighed by the inclusion of a second $\mathrm{Cu}$ atom.

Although the calculations reveal the favorable formation of metal-coordination bonds to $\mathrm{Cu}$ adatoms, they still do not fully explain the experiment. We noted a strict 
a)
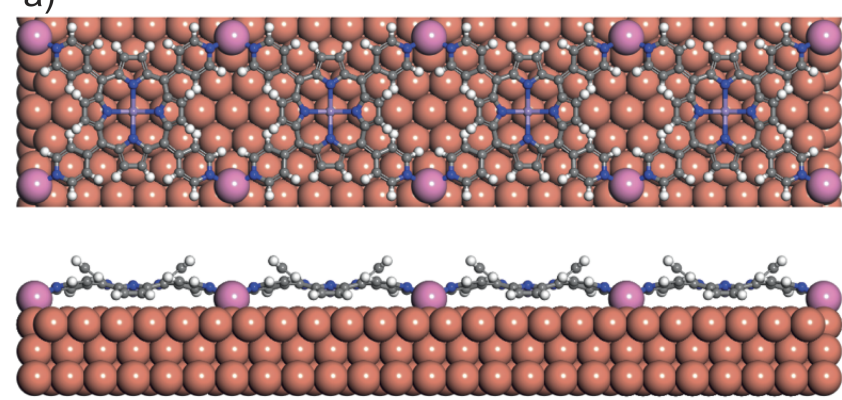

b)
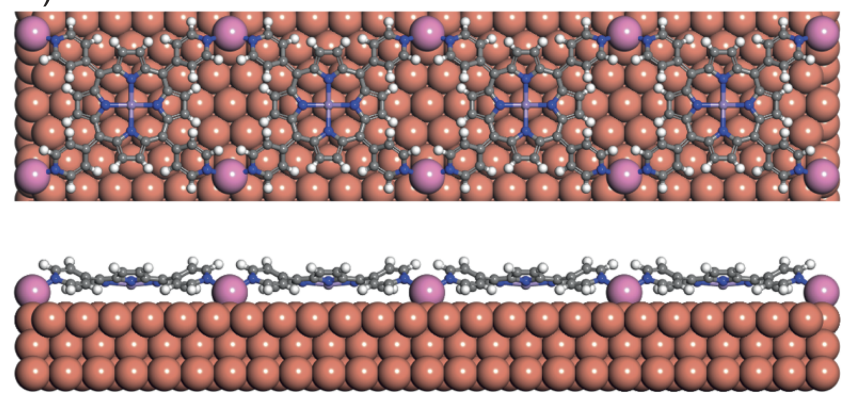

c)
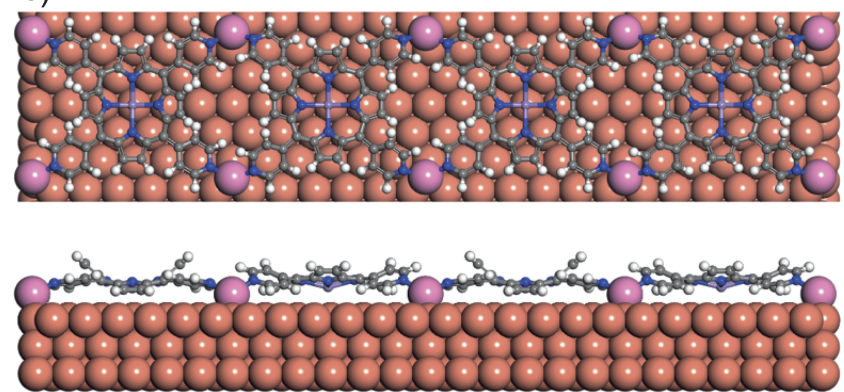

FIG. 4. Top and side views of the structure-optimized periodic chains of Mn-TPyP and $\mathrm{Cu}$ adatoms. (a) Chain of type A molecules on top sites with $\mathrm{Cu}$ atoms on hollow sites along the $\langle 1 \overline{1} 0\rangle$ direction. (b) Chain of type $\mathrm{B}$ molecules on top sites with $\mathrm{Cu}$ atoms on hollow sites along the $\langle 1 \overline{1} 0\rangle$ direction. (c) Chain of alternating type A and type B molecules on top sites with $\mathrm{Cu}$ atoms on hollow sites along the $\langle 1 \overline{1} 0\rangle$ direction.

alternation of type A and type B molecules if only one $\mathrm{Cu}$ atom is located between the pyridyl rings. To unravel the origin of this pattern, we carry out calculations on the alternating structure (for a model, see Fig. 4(c)). We find that the adsorption energy in these mixed chains is increased compared to the pure type $\mathrm{A}-\mathrm{Cu}$ or pure type $\mathrm{B}-\mathrm{Cu}$ chains. A close inspection of the molecular structures suggests that steric repulsion is the origin of the lower adsorption energy of the homo-type metal-organic chains. In the homo-molecular chains, the pyridyl rings of neighboring

TABLE II. Adsorption energies and bond lengths from $\mathrm{PBE}+\mathrm{D} 2+\mathrm{U}$ calculations of type $\mathrm{A}$ and type $\mathrm{B}$ molecules and their mixture in metal-organic chains on top sites of $\mathrm{Cu}(111)$. Adsorption energies refer to the adsorption energy of the molecules only, i.e., $\mathrm{E}_{\mathrm{ads}}=\mathrm{E}_{\mathrm{total}}-\left(\mathrm{E}_{\mathrm{Cu}(111)+\mathrm{Cu}_{\mathrm{ad}}}+\mathrm{E}_{\mathrm{mol}}\right)$. Energies are given in $\mathrm{eV}$ and bond lengths in $\AA$.

\begin{tabular}{lrrc}
\hline \hline & $\mathrm{A}-\mathrm{Cu}$ & $\mathrm{B}-\mathrm{Cu}$ & $\mathrm{A}-\mathrm{Cu}-\mathrm{B}-\mathrm{Cu}$ \\
\hline $\mathrm{E}_{\text {ads }}$ & -7.20 & -5.42 & -15.34 \\
$D_{\mathrm{Cu}_{\text {sub }}}-\mathrm{Mn}$ & 2.54 & 2.76 & $2.59(\mathrm{~A})$ and $2.67(\mathrm{~B})$ \\
$D_{\mathrm{Cu}_{\mathrm{ad}}}-\mathrm{N}$ & 2.12 & 2.14 & $2.08(\mathrm{~A})$ and $2.06(\mathrm{~B})$ \\
\hline \hline
\end{tabular}

TABLE III. Deformation angles according to Fig. 1 (in deg) from $\mathrm{PBE}+\mathrm{D} 2+\mathrm{U}$ calculations of Mn-TPyP molecules on $\mathrm{Cu}(111)$ and in the metal-organic chains obtained after relaxation.

\begin{tabular}{lcclc}
\hline \hline & $\rho_{1}$ & $\rho_{2}$ & $\theta$ & $\phi$ \\
\hline $\mathrm{A}$ & 23.2 & -13.9 & 31.6 & -6.0 \\
$\mathrm{~B}$ & -15.3 & 26.2 & 28.1 & -4.9 \\
$\mathrm{~A}-\mathrm{Cu}$ & 38.2 & -17.2 & 13.2 & 4.8 \\
$\mathrm{~B}-\mathrm{Cu}$ & -12.4 & 19.1 & 28.8 & 2.3 \\
$\mathrm{~A}-\mathrm{Cu}-\mathrm{B}-\mathrm{Cu}$ & $40.8(\mathrm{~A})$ & $-17.1(\mathrm{~A})$ & $10.7(\mathrm{~A})$ & $2.9(\mathrm{~A})$ \\
& $-16.0(\mathrm{~B})$ & $21.7(\mathrm{~B})$ & $26.8(\mathrm{~B})$ & $3.9(\mathrm{~B})$ \\
& & & &
\end{tabular}

molecules are tilted towards each other. For the type $\mathrm{A}-\mathrm{Cu}$ chain, this leads to a closest hydrogen-hydrogen distance of $1.62 \AA$ and for the type $\mathrm{B}-\mathrm{Cu}$ chain to a closest distance of $1.78 \AA$. These two values are significantly smaller than twice the van der Waals radius of hydrogen $(2.4 \AA)$. Such close proximity would result in strong repulsive forces, thus inhibiting the occurrence of these structures. For the mixed metal-organic chain, the nearest hydrogen-hydrogen-distance is much larger $(2.32 \AA)$ and indicates that there is nearly no steric repulsion between the pyridyl legs. A similar conclusion has been proposed from purely experimental observations of free-base TPyP-Fe chains. ${ }^{19}$ To validate the obtained $\mathrm{A}-\mathrm{Cu}-\mathrm{B}-\mathrm{Cu}$ chain structures from DFT against experiment, we have simulated the frequency shift images obtained with a CO tip with the simulation program of Hapala and coworkers. ${ }^{48}$ This simulation is based on the repulsive forces between the flexible $\mathrm{CO}$ termination of the tip and the molecules on the surface. ${ }^{46,47}$ The agreement for both type A and type B molecules corroborates our theoretical findings (compare Figs. 3(b) and 3(c)).

Our experiments and calculations thus draw a complete picture of the adsorption scenario of Mn-TPyP on $\mathrm{Cu}(111)$. The metal-organic structure with two types of molecules is a result of the energy balance from inter- and intramolecular interactions and bond formation. The many degrees of freedom bear the advantage of a versatile adaptation of the porphyrins to their atomic-scale environment. The drawback of this flexibility is the difficulty in controlling the structure. Small variations in temperature or packing density may lead to different structures.

\section{Electronic properties}

We now investigate whether the electronic structure strongly differs in the two molecular conformations. The different appearance in STM images may not only be linked to the topography of the molecule, but it also includes the electronic structure. Hence, molecules may appear much brighter or darker than anticipated from the conformation. We perform tunneling spectroscopy on the two types of molecules. Differential conductance $(\mathrm{d} I / \mathrm{d} V)$ spectra taken on the pyridyl endgroups of type A molecules exhibit a broad feature at $2.7 \mathrm{~V}$. This indicates a strong electronic coupling of the molecular states with the substrate states. This observation agrees with the flattened pyridyl rings. In contrast, type B molecules show three separate resonances at positive bias voltages up to $3 \mathrm{~V}$ (Figure 5). These peaks are located at $0.4 \mathrm{~V}, 1.4 \mathrm{~V}$, 

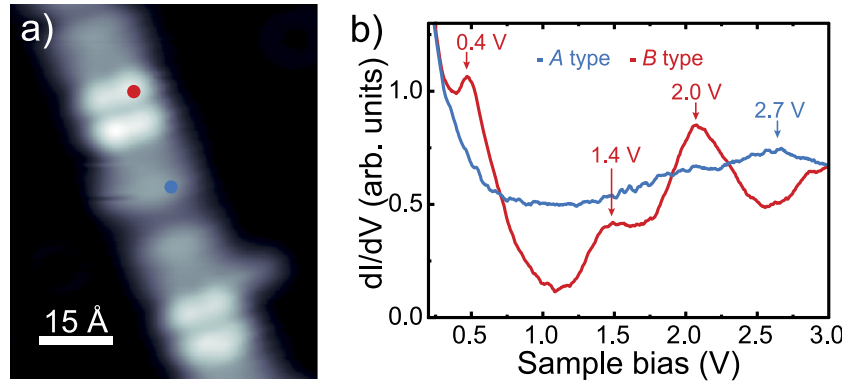

FIG. 5. (a) STM image with indicated locations of the $\mathrm{d} I / \mathrm{d} V$ spectra $(V=1 \mathrm{~V}, I=100 \mathrm{pA})$. (b) $\mathrm{d} I / \mathrm{d} V$ spectra on type A molecules show a broad resonance and spectra on type $\mathrm{B}$ molecules show three sharper resonances (recorded at constant current $I=50 \mathrm{pA}$ ).

and $2.0 \mathrm{~V}$. The sharper resonances suggest a weaker electronic coupling to the substrate. This is in agreement with the pyridyl rings being tilted further away from the substrate and a larger $\mathrm{Cu}-\mathrm{Mn}$ distance. We note that we do not observe clear resonances at negative bias voltages up to $-3 \mathrm{~V}$ in both molecular types.

We have calculated the density of states (DOS) for type A and type B molecules with adatoms (see Fig. 6). The conduction bands, which would correspond to the low-lying LUMOs, occur in the same energy range as the measured resonances. The dominant contribution in the DOS originates from the carbon atoms. Type A shows weak peaks up to $2 \mathrm{eV}$, which are not separately resolved in the experiment. An intensive broad peak occurs slightly above $2 \mathrm{eV}$ which could correspond to the broad resonance at $2.7 \mathrm{eV}$ in experiment. For type B there are four peaks of similar height. The first one agrees well with the measured one at $0.4 \mathrm{eV}$. The shoulder at $1.4 \mathrm{eV}$ in experiment can be attributed to the second peak in the calculated DOS at about $1 \mathrm{eV}$. Most probably the two largest peaks at $1.6 \mathrm{eV}$ and $2 \mathrm{eV}$ combine to the pronounced resonance at $2 \mathrm{eV}$ in experiment. All the calculated resonances (for both types of molecules) are at somewhat lower energy values than the measured ones. DFT-GGA methods, such as the used PBE functional, typically underestimate the size of energy gaps between occupied and unoccupied states (see, e.g., Ref. 49). This is particularly true for the HOMO-LUMO gap in molecules. In view of this deficiency, the comparison between theory and experiments is reasonably good and supports the interpretation of the molecular structures.

\section{Conformational changes induced by STM manipulation}

Having resolved the important structural and electronic properties of the two molecular types, we try to convert a)

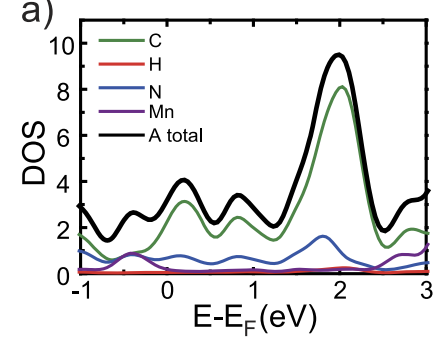

b)

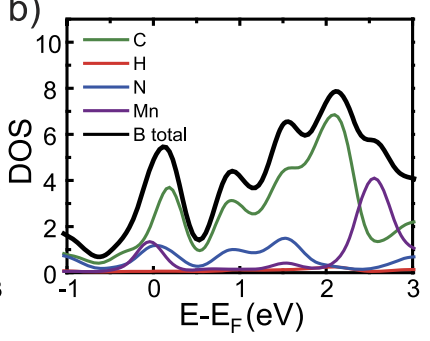

FIG. 6. (a) Projected density of states on the atoms of type A and type B molecules in the A-Cu (a) and B-Cu (b) chains.

the molecular conformations with the STM tip. We first try the manipulation on chain segments, which only include type A molecules, i.e., those which have two $\mathrm{Cu}$ adatoms included between the nearest neighbors (see Fig. 7). Placing the tip on one of the pyridyl rings and ramping up the bias voltage while keeping the current constant reveal a sudden increase in tip height at $2.6 \mathrm{~V}$. This change signifies a conformational change of the molecule. The simultaneously recorded differential conductance signal undergoes a sudden jump at the same time. Both z- and dI/dV-signals remain stable upon further increase of the bias voltage up to $3 \mathrm{~V}$. Reducing the bias voltage does not lead to sudden changes, thus signifying that the molecule is in a stable state. Whereas the tip position is higher, which agrees with a larger height of the pyridyl ring, the $\mathrm{dI} / \mathrm{dV}$ signal exhibits more pronounced resonances on the reverse bias scan. Posterior imaging of the same chain reveals that the addressed molecule exhibits two brighter legs. A differential conductance spectrum recorded on the bright leg reveals the resonance of type B molecules (see above), whereas the dark legs show the typical broad feature of type A molecules. Hence, we conclude that the pyridyl legs have been rotated around their axis, now exhibiting a steeper inclination angle $\theta$. Although we cannot determine the precise value of the angle, we suspect that it is similar to the value of $\theta=26.8^{\circ}$ of type B molecules because the differential conductance signals are similar. We attempted the activation of the rotation process as described above on several molecules. The minimum voltage for the switching is $2.0 \mathrm{eV}$, which matches the onset of resonances for type $\mathrm{A}$ and type B molecules and hints at a resonantly enhanced mechanism. We have never observed a back-rotation of the pyridyl moiety, also not at negative bias voltage or higher current density.

We found that switching of type A molecules is also possible in the mixed chains. Figure 8 shows a series of such events induced by the STM tip. We start by placing the tip on a pyridyl moiety of a type A molecule as indicated in Fig. 8(a).
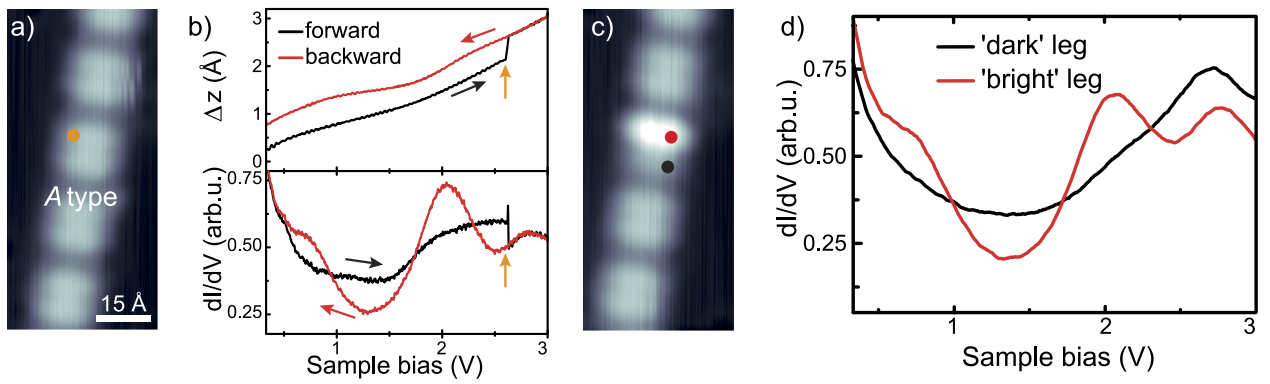

FIG. 7. Rotation of pyridyl legs in A$\mathrm{Cu}-\mathrm{Cu}$ chains. (a) STM image of the chain before and (c) after ramping up the bias to $3 \mathrm{~V}(\mathrm{~V}=1 \mathrm{~V}, \mathrm{I}=110 \mathrm{pA})$. (b) $\mathrm{z}-\mathrm{V}$ - and $\mathrm{dI} / \mathrm{dV}-\mathrm{V}$ spectra during the manipulation process with feedback on (setpoint: $\mathrm{V}=0.2 \mathrm{~V}, \mathrm{I}=0.2 \mathrm{nA}$ ). (d) $\mathrm{dI} / \mathrm{dV}$ spectra acquired on the intact and rotated pyridyl legs with feedback on (setpoint: $\mathrm{V}=0.2 \mathrm{~V}, \mathrm{I}=0.2 \mathrm{nA}$ ). 

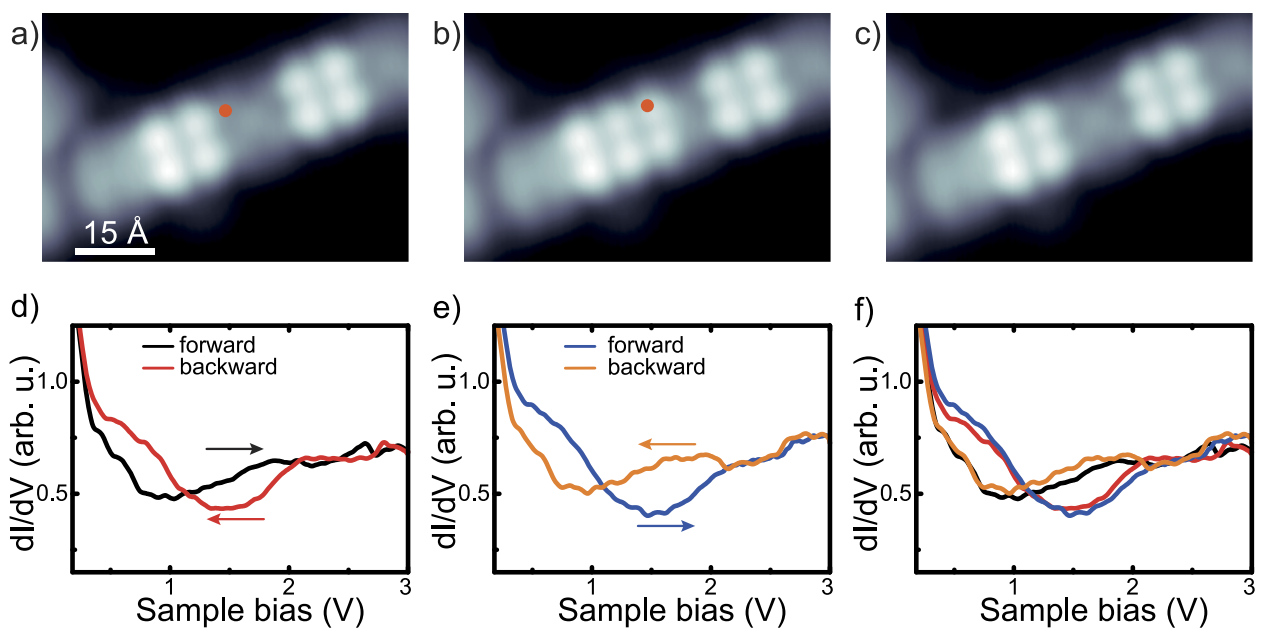

FIG. 8. Reversible rotation of pyridyl legs of type A molecules in $\mathrm{A}-\mathrm{Cu}-\mathrm{B}-$ $\mathrm{Cu}$ chains. STM image of the chain before (a), after one (b), and after two (c) switching events $(\mathrm{V}=1 \mathrm{~V}, \mathrm{I}=0.1$ $\mathrm{nA}$ ). The rotation was induced by sweeping the bias voltage during the spectra in (d) and (e) while recording dI/dV spectra with feedback on (setpoint: $\mathrm{V}=0.1$ $\mathrm{V}, \mathrm{I}=50 \mathrm{pA}$ ). (f) Superposition of the $\mathrm{dI} / \mathrm{dV}$ spectra in (d) and (e) showing the reversibility of the process.
Recording a dI/dV spectrum at constant current while increasing the bias voltage reveals the broad feature, whereas the backward sweep shows a clear separation of resonances below $1 \mathrm{eV}$ and above $2 \mathrm{eV}$. Despite the poorer data quality, we find the same characteristics as the type A and type B molecules, respectively. A consecutively recorded STM image reveals brighter contrast of two pyridyl moieties, in agreement with the $\mathrm{dI} / \mathrm{dV}$ signal. When applying the same bias voltage ramp again, we observe a back rotation of the pyridyl rings. This is clearly reflected when superimposing the spectra (Fig. 8(f)). Posterior STM imaging also reflects the reversible rotation of the pyridyl moiety around the angle $\theta$ into its original position.

The reversibility of the rotation process with the same parameters suggests that the reaction mechanism and barrier for the two processes are similar. We identified that the mixture of type A and type B molecules is enforced by intermolecular steric repulsion. The ability to rotate the pyridyl against the favored direction requires some energy, but back-switching is possible to end up in the energetically favored state. This is in strong contrast to the behavior observed in the pure type A chains with two $\mathrm{Cu}$ adatoms within the molecules, where we could not observe the back-rotation in our parameter range.

\section{CONCLUSIONS}

We have identified two types of molecular conformations of Mn-TPyP on a Cu(111) surface. Combining STM and AFM experiments with DFT calculations, we could resolve the molecular structures. We showed that the driving force for the molecular distortions is the formation of adatom-pyridyl bonds. The pattern formation of alternating molecular conformers with single $\mathrm{Cu}$ adatoms is enforced by intermolecular repulsion. These results highlight the flexibility of the porphyrins to adapt to their environment. The differential conductance spectra of the conformers differ in two types, probably as a result of different hybridization strength with the substrate due to the different inclination angles of the pyridyl moieties. By applying voltage ramps, we could induce rotations of the pyridyl legs. In most cases, not only the pyridyl moiety directly underneath the tip but also its neighbor undergoes the same rotation. The ability to induce reversible conformational changes differs significantly in the different environments. This emphasizes the crucial role of interactions with the atomic neighborhood, which determines the energy landscape. In view of the vision of molecular electronics, our results stress the need of a detailed knowledge of the device environment at the atomic scale. Small changes already change the energy balance of states and favor certain molecular states over others. As a result, their electronic properties, stability, and addressing ability are strongly influenced.

\section{ACKNOWLEDGMENTS}

We thank the Deutsche Forschungsgemeinschaft for funding through Sonderforschungsbereich 658. X.C. acknowledges funding by the Chinese Scholarship Council (CSC).

${ }^{1}$ T. Yokoyama, S. Yokoyama, T. Kamikado, Y. Okuno, and S. Mashiko, Nature 413, 619 (2001).

${ }^{2}$ N. Wintjes, D. Bonifazi, F. Cheng, A. Kiebele, M. Stöhr, T. Jung, H. Spillmann, and F. Diederich, Angew. Chem., Int. Ed. 46, 4089 (2007).

${ }^{3}$ D. Ecija, K. Seufert, D. Heim, W. Auwärter, C. Aurisicchio, C. Fabbro, D. Bonifazi, and J. V. Barth, ACS Nano 4, 4936 (2010).

${ }^{4}$ W. Auwärter, D. Écija, F. Klappenberger, and J. V. Barth, Nat. Chem. 7, 105 (2015).

5 J. M. Gottfried, Surf. Sci. Rep. 70, 259 (2015).

${ }^{6}$ D. Heim, K. Seufert, W. Auwärter, C. Aurisicchio, C. Fabbro, D. Bonifazi, and J. V. Barth, Nano Lett. 10, 122 (2010).

${ }^{7}$ D. Heim, E. David, K. Seufert, W. Auwärter, D. Heim, E. David, C. Fabbro, D. Bonifazi, and J. V. Barth, J. Am. Chem. Soc. 132, 6783 (2010).

${ }^{8}$ Y. Li, J. Xiao, T. E. Shubina, M. Chen, Z. Shi, M. Schmid, H.-P. Steinrück, J. M. Gottfried, and N. Lin, J. Am. Chem. Soc. 134, 6401 (2012).

${ }^{9}$ Y. Li and N. Lin, Phys. Rev. B 84, 125418 (2011).

${ }^{10}$ D. Ecija, M. Marschall, J. Reichert, A. Kasperski, D. Nieckarz, P. Szabelski, W. Auwärter, and J. Barth, Surf. Sci. 643, 91 (2016).

${ }^{11}$ F. Bischoff, Y. He, K. Seufert, D. Stassen, D. Bonifazi, J. V. Barth, and W. Auwärter, Chem. - Eur. J. 22, 15298 (2016).

${ }^{12}$ W. Auwärter, A. Weber-Bargioni, A. Riemann, A. Schiffrin, O. Gröning, R. Fasel, and J. V. Barth, J. Chem. Phys. 124, 194708 (2006).

${ }^{13}$ W. Auwärter, F. Klappenberger, A. Weber-Bargioni, A. Schiffrin, T. Strunskus, C. Wöll, Y. Pennec, A. Riemann, J. V. Barth, and D. Bochum, J. Am. Chem. Soc. 129, 11279 (2007).

${ }^{14}$ W. Auwärter, A. Weber-Bargioni, S. Brink, A. Riemann, A. Schiffrin, M. Ruben, and J. V. Barth, ChemPhysChem 8, 250 (2007).

${ }^{15}$ J. P. Jarvis, S. Taylor, J. D. Baran, D. Thompson, A. Saywell, B. Mangham, N. R. Champness, J. A. Larsson, and P. Moriarty, J. Phys. Chem. C 119, 27982 (2015).

${ }^{16}$ F. Klappenberger, A. Weber-Bargioni, W. Auwärter, M. Marschall, A. Schiffrin, and J. V. Barth, J. Chem. Phys. 129, 214702 (2008).

${ }^{17}$ Z. Shi and N. Lin, J. Am. Chem. Soc. 131, 5376 (2009). 
${ }^{18}$ G. Lyu, R. Zhang, X. Zhang, P. Nian Liu, and N. Lin, J. Mater. Chem. C 3, 3252 (2015).

${ }^{19}$ Y. Wang, Z. Kun, Z. Shi, and Y.-Q. Ma, Phys. Chem. Chem. Phys. 18, 14273 (2016).

${ }^{20}$ V. Iancu, A. Deshpande, and S.-W. Hla, Nano Lett. 6, 820 (2006).

${ }^{21}$ J. Brede, M. Linares, R. Lensen, A. E. Rowan, M. Funk, M. Bröring, G. Hoffmann, and R. Wiesendanger, J. Vac. Sci. Technol., B 27, 799 (2009).

${ }^{22}$ J. P. Jarvis, S. Taylor, J. D. Baran, N. R. Champness, J. A. Larsson, and P. Moriarty, Nat. Commun. 6, 8338 (2015).

${ }^{23}$ F. Moresco, G. Meyer, K.-H. Rieder, H. Tang, A. Gourdon, and C. Joachim, Phys. Rev. Lett. 86, 672 (2001).

${ }^{24}$ X. H. Qiu, G. V. Nazin, and W. Ho, Phys. Rev. Lett. 93, 196806 (2004).

${ }^{25}$ S. Ditze, M. Stark, F. Buchner, A. Aichert, N. Jux, N. Luckas, A. Görling, W. Hieringer, J. Hornegger, H.-P. Steinrück, and H. Marbach, J. Am. Chem. Soc. 136, 1609 (2014).

${ }^{26}$ F. Albrecht, F. Bischoff, W. Auwärter, J. V. Barth, and J. Repp, Nano Lett. 16, 7703 (2016).

${ }^{27}$ A. E. Mattsson, R. Armiento, J. Paier, G. Kresse, J. M. Wills, and T. R. Mattsson, J. Chem. Phys. 128, 084714 (2008).

${ }^{28}$ G. Kresse and J. Furthmüller, Comput. Mater. Sci. 6, 15 (1996).

${ }^{29}$ G. Kresse and J. Furthmüller, Phys. Rev. B 54, 11169 (1996).

${ }^{30}$ J. P. Perdew, K. Burke, and M. Ernzerhof, Phys. Rev. Lett. 77, 3865 (1996).

${ }^{31}$ S. Grimme, J. Comput. Chem. 27, 1787 (2006).

${ }^{32}$ K. Leung, S. B. Rempe, P. A. Schultz, E. M. Sproviero, V. S. Batista, M. E. Chandross, and C. J. Medforth, J. Am. Chem. Soc. 128, 3659 (2006).

${ }^{33}$ Z. Shi, J. Liu, T. Lin, F. Xia, P. N. Liu, and N. Lin, J. Am. Chem. Soc. 133, 6150 (2011).

${ }^{34}$ T. R. Umbach, M. Bernien, C. F. Hermanns, L. L. Sun, H. Mohrmann, K. E. Hermann, A. Krüger, N. Krane, Z. Yang, F. Nickel, Y.-M. Chang, K. J. Franke, J. I. Pascual, and W. Kuch, Phys. Rev. B 89, 235409 (2014).
${ }^{35}$ S. Clair, S. Pons, H. Brune, K. Kern, and J. V. Barth, Angew. Chem., Int. Ed. 44, 7294 (2005).

${ }^{36}$ S. Clair, S. Pons, S. Fabris, S. Baroni, H. Brune, K. Kern, and J. V. Barth, J. Phys. Chem. B 110, 5627 (2006).

${ }^{37}$ S. Stepanow, N. Lin, D. Payer, U. Schlickum, F. Klappenberger, G. Zoppellaro, M. Ruben, H. Brune, J. V. Barth, and K. Kern, Angew. Chem., Int. Ed. 46, 710 (2007).

${ }^{38}$ U. Schlickum, R. Decker, F. Klappenberger, G. Zoppellaro, and S. Klyatskaya, Nano Lett. 7, 3813 (2007).

${ }^{39}$ J. Björk, M. Matena, M. Dyer, M. Enache, J. Lobo-Checa, L. Gade, T. Jung, M. Stöhr, and M. Persson, Phys. Chem. Chem. Phys. 12, 8815 (2010).

${ }^{40}$ N. Henningsen, R. Rurali, C. Limbach, R. Drost, J. I. Pascual, and K. J. Franke, J. Phys. Chem. Lett. 2, 55 (2011).

${ }^{41}$ T. Classen, M. Lingenfelder, Y. Wang, R. Chopra, C. Virojanadara, U. Starke, G. Costantini, G. Fratesi, S. Fabris, S. De Gironcoli, S. Baroni, S. Haq, R. Raval, and K. Kern, J. Phys. Chem. A 111, 12589 (2007).

${ }^{42}$ Z. Yang, M. Corso, R. Robles, C. Lotze, R. Fitzner, E. Mena-Osteritz, P. Bäuerle, K. J. Franke, and J. I. Pascual, ACS Nano 8, 10715 (2014).

${ }^{43}$ T. Classen, G. Fratesi, G. Costantini, S. Fabris, F. L. Stadler, C. Kim, S. de Gironcoli, S. Baroni, and K. Kern, Angew. Chem., Int. Ed. 44, 6142 (2005).

${ }^{44}$ S. Haq, F. Hanke, M. S. Dyer, M. Persson, P. Iavicoli, D. B. Amabilino, and R. Raval, J. Am. Chem. Soc. 133, 12031 (2011).

${ }^{45}$ L. Gross, F. Mohn, N. Moll, P. Liljeroth, and G. Meyer, Science 325, 1110 (2009).

${ }^{46}$ P. Hapala, G. Kichin, C. Wagner, F. S. Tautz, R. Temirov, and P. Jelínek, Phys. Rev. B 90, 085421 (2014).

${ }^{47}$ P. Hapala, R. Temirov, F. S. Tautz, and P. Jelínek, Phys. Rev. Lett. 113, 226101 (2014).

${ }^{48}$ Probe Particle Model, accessed September 2016.

${ }^{49}$ Y. Zhao and D. G. Truhlar, J. Chem. Phys. 130, 074103 (2009). 